\title{
LENGUA Y CULTURA EN LAS CANCIONES DE ARMA
}

\author{
Isabel Gálvez Astorayme
}

Sumilla:

Teniendo como base su experiencia de campo en la comunidad de Ama, departamento de Huancavelica, la lingüista Isabel Gálvez nos muestra la estrecha relación que existe entre Lengua, Sociedad y Cultura, a partir del análisis de dos canciones quechuas, "Ira" y "Sillkaw".

\section{Abstract:}

With their fieldwork experiences the author in the Ama Community, the linguist Isabel Galvez, show us the close relationship between language, society and culture, since an analysis of two quechua songs, "Ira" and "Sillkaw".

Junto a las modalidades para la producción de medios de subsistencia, la población campesina asume formas de pensar y sentir, tanto los elementos de su medio ambiente como las actividades con que las encara, el aprovechamiento de los recursos. Todo esto, que constituye parte de la cultura de una sociedad ligada fundamentalmente al agro, es una constante en la interacción cotidiana de sus miembros, y en ella juega papel importante el idioma. En ese sentido, el idioma se ve convertido en un factor de autoidentificación del grupo que es percibido, frente a cualquier otro, como el que mejor expresa los sentimientos del ánimo, las cosas de la tierra y de la vida. Bajo estas condiciones, el idioma puede convertirse en uno de los elementos fundamentales para la conservación, adquisición o readquisición de una conciencia nacional, cuando coadyuvan para ello factores extralingüísticos, básicamente económicos y políticos (Torero,1974:70).

En efecto, una de las formas de sentir y de pensar del grupo social que integra la comunidad de Arma, se manifiesta en sus canciones en lengua quechua entonadas en el Waylas; danza agraria que se realiza al término de las faenas agrícolas del día; en ellas se expresa la pérdida de sus valores culturales o su situación de marginación social, por imposición histórica de lengua y cultura dominante, porque los fenómenos lingüísticos que se originan por contacto de lenguas no están exentos de provocar problemas de tipo social, ya que cuando dos o más lenguas conviven en una comunidad, una de ellas tiende a ser dominante (Sara Bolaños, 1982:68). En suma, se advierte que este dominio tiene raíces históricas y sociales, pues no se trata que una lengua sea major o más idónea que otra.

La comunidad de Arma está ubicada en el distrito del mismo nombre, en la provincia de
Castrovirreyna, al Sureste del departamento de Huancavelica, entre 2200 y 5032 metros sobre el nivel del mar, a 101,30 kilómetros de la provincia de Chincha. En los linderos del distrito yacen los restos arqueológicos de Warmillaqta, Tawrimarka y Tunsuwillka.

\section{ASPECTO LINGÜÍSTICO}

En la comunidad de Arma se habla el quechua y el castellano. El quechua de Arma pertenece a la variedad dialectal ayacuchana, que corresponde al área dialectal del Quechua II-C (Torero, 1964). Sin embargo, Arma maneja algunos lexemas no usados por el resto de la variedad dialectal ayacuchana (Gálvez, 1985:7), como por ejemplo:

\begin{tabular}{|c|c|c|}
\hline Glosa & ARMA & AYACUCF \\
\hline ¿cuándo? & ¿imay? & ¿haykap? \\
\hline enjuagarse la boca & muqchi & chumchi \\
\hline picaflor & qinchu & qinti \\
\hline lana & millwa & millma \\
\hline lugar solitario & chunyaq & chunni \\
\hline flor de maíz & pawaru & parwa \\
\hline hormiga & añayllu & sisi \\
\hline seso & piqa & ñutqu \\
\hline pulmón & sunqu & qapsan \\
\hline
\end{tabular}

Siendo el quechua la lengua materna de los habitantes de la zona, el castellano se usa con interferencias de la lengua materna, las que pueden llegar a ser más o menos notorias según el grado de bilingüismo del hablante. A su vez, el castellano ha llegado a influir sobre el quechua, pues no es poco frecuente que en el 
uso del quechua se recurra a préstamos del castellano, cuando se trata de conceptos que por ser extraños a la cultura nativa carecen de significantes en el quechua. Estos préstamos lingüísticos suelen ser quechuizados mediante su ligazón con uno o más morfemas quechuas o mediante la adaptación al fonetismo quechua.

Estos fenómenos lingüísticos se observan en las canciones Ira y Sillkaw, que hemos escogido de la variedad de canciones de la comunidad de Arma, entonadas en quechua durante la noche del waylas. Al mismo tiempo, ambas revelan la imposición del mercantilismo y la marginación social, respectivamente.

Ira es la pronunciación que el quechuahablante de la zona hace del término castellano "era", que en el castellano general nombra al espacio de tierra limpia y firme, algunas veces empedrado, donde se trillan las mieses y en el castellano de la zona, al espacio para reunir transitoriamente la cosecha de papa, maíz, trigo, etc.

En los fonemas / e, o / de vocablos castellanos suele darse con frecuencia la interferencia vocálica, porque en el sistema vocálico del quechua no existen tales fonemas, sino las vocales altas $/ \mathrm{i}, \mathrm{u} /$. De allí que ira provenga de era.

Cuando se refiere a este tipo de interferencia, Cerrón-Palomino (1975:132-134) señala que ello se produce cuando el bilingüe identifica los fonemas de la segunda lengua con los de la materna. Dicha identificación es automática y está determinada, entre otras cosas, por los hábitos articulatorios del bilinguie.

En la canción Ira, que se canta mediante un diálogo entre un coro de mujeres (M) y otro de hombres $(\mathrm{H})$, la transferencia léxica y la transferencia fonológica trascienden al título de la canción:

M Ñachum ira kichasqaña, ¿Ya está la era preparada, sawanay kullkuy,

kurdubina papa yaykunanpaq, sawanay kullkuy. sawanay kullkuy, para que entre la papa cordovina, sawanay kullkuy?

H. Ñam ira kichasqaña, sawanay kullkuy, kurdubina papa yaykunanpaq, sawanay kullkuy.

Ya está la era preparada, sawanay kullkuy, para que entre la papa sawanay kullkuy. cordovina,

Sawanay kullkuy es una frase que aparece en la canción como un estribillo. Literalmente significa "tener que cargar mi tortolita".

En la segunda parte de la misma canción, tales tipos de transferencia se encuentran también presentes, como por ejemplo cuando los hombres responden al desafío de las mujeres:

H. Kananmi sawasqayki, sawanay kullkuy, kaballiru usuchata, sawanay kullkuy.

Ahora mismo te cargaré, sawanay kullkuy, a la manerita del caballero, sawanay kullkuy.

O cuando las mujeres siguen desafiando a los hombres:

M ¿Ima sawawankimanraq, ¿Cómo me podrías cargar, sawanay kullkuy, barritiru makiykiwan, sawanay kullkuy, sawanay kullkuy. con tu mano de barretero, sawanay kullkuy.

En la canción Sillkaw -que igualmente es un diálogo entre un coro de mujeres y otro de hombrestambién se observa las transferencias fonológicas y lexicales, clando en la canción las mujeres responden:

M Manam, wawaytaqa, sillkaw sillkaw. Mi hija no, ... Mayistrapaqmi, sillkaw sillkaw. Es para maestra,... Dukturapaqmi, sillkaw sillkaw. Es para doctora,... Abugadapaqmi, sillkaw sillkaw. Es para abogada,... Midikupaqmi, sillkaw sillkaw. Es para médico,...

Sillkaw es nombre de un arbusto cuyo fruto seco, en forma de pequeños filamentos, está coronado por unas espinitas dispuestas en forma de estrella que tienen la particularidad de adherirse a la ropa al hacerse contacto con ellas. La repetición de este nombre en la canción es un estribillo. En la zona de Arma, como en toda la cultura andina, para los estribillos suele recurrirse a nombres de especies de la flora o de la fauna.

Tanto en la canción Ira como en la canción Sillkaw encontramos, pues, transferencias de palabras castellanas al léxico quechua, en las cuales se opera la transferencia fonológica:

era $<$ ira
cordovina $<$ kurdubina
caballero < kaballiru
barretero $<$ barritiru
maestro $<$ mayistru
doctor $<$ duktur
abogado $<$ abugadu
enfermera $<$ infirmira
médico < midiku
arquitecto $<$ arkitecto

En situaciones de contacto son innumerables los ejemplos de transferencia o préstamos de palabras y morfemas radicales de una lengua a otra. Los préstamos pueden consistir en la transferencia de item léxico o lexema de una lengua (Silva-Corvalán, 1989:171). Ésto se corrobora en los hablantes de la comunidad de Arma, cuando los lexemas castellanos han sido quechuizados 
mediante el cambio y la adaptación fonética y por la ligazón de un morfema, en este caso el morfema benefactivo -paq "para", como en:

$\begin{array}{ll}\text { Mayistrupaqmi. } & \text { 'Es para maestro'. } \\ \text { Abugadupaqmi. } & \text { 'Es para abogado'. } \\ \text { Infirmirapaqmi. } & \text { 'Es para enfermera'. }\end{array}$

\section{ASPECTOCULTURAL}

Como se ha dicho, una de las formas de pensar y sentir del grupo social que integra la comunidad de Arma, -grupo eminentemente agrario- se manifiesta en las canciones que cantan en el waylas, durante el proceso de la cosecha. "La producción agrícola liga al trabajo, al amor, la alegría. Por eso, el canto y la danza acompañan las faenas" (Montoya, 1987: 28).

El waylas es una danza agraria que bailan hombres y mujeres, al término de las faenas agrícolas, al son de canciones -en quechua- picarescas y satíricas, 0 canciones de lamento por la pérdida de sus valores culturales. El waylas se realiza durante el tiempo de cosecha en las tierras comunales, en los meses de mayo y junio, por las noches, después de haber terminado la faena del día.

La organización de la fiesta corre a cargo de un grupo de personas o de una familia que ya tiene preparada la era a un extremo del terreno cosechado. Los organizadores invitan a las familias vecinas, especialmente a las que cuentan con jóvenes de uno y otro sexo. La cantidad de participantes es numerosa, pero últimamente sólo la gente adulta practica esta actividad.

Para iniciar el baile hay un personaje que dirige la fiesta. Se entonan canciones que describen la situación socio-cultural y económica de la comunidad. A veces describen la evolución del cultivo en general, otras veces expresan con humor su crítica a las autoridades. El waylas suele iniciarse a las seis de la tarde y concluir a las cuatro o cinco de la madrugada.

En la canción Ira expresan la añoranza por las variedades de papas que ya no se cultivan en la comunidad, o están en vía de extinción, como consecuencia del mercantilismo que ha introducido nuevas variedades que logran resultados en menor tiempo o rinden mayor cantidad, pero que son de calidad inferior a los de las variedades nativas desplazadas.

Esto indica que por influencia del Mercantilismo y la falta de orientación tecnológica, los comuneros optaron por reemplazar los productos nativos de la localidad por productos comerciales de la costa, de calidad inferior como la papa Renacimiento, Tomasa, etc.

La canción es un diálogo entre un coro de mujeres que pregunta y un coro de hombres que responde:

M Ñachum ira kichasqaña, ¿Ya está la era preparada, sawanay kullkuy, sawanay kullkuy, Kunyaq papa, para que entre la papa yaykunanpaq, kunyaq, sawanay kullkuy. sawanay kullkuy?

H Ñam ira kichasqaña, sawanay kullkuy, kunyaq papa yaykunanpaq, sawanay kullkuy.

Ya está la era preparada, sawanay kullkuy, para que entre la papa sawanay kullkuy. kunyaq,

En este diálogo vemos la añoranza que sienten los campesinos frente a la papa Kunyaq, que es una papa arenosa de sabor agradable, de cáscara negruzca y de almidón amarillo, parecida a la papa Huairo. Esta variedad ha desaparecido de la zona.

Las preguntas y respuestas se suceden, enumerando distintas variedades de papas, como se indica en la siguiente relación, extraída de la canción mencionada:

Kunyaq

Kurdubina

Yana paltas

Aqu suytu

Qala suytu

Misipa makin

Qillu runtus
Papa arenosa de sabor muy agradable, de cáscara negruzca y de almidón amarillo, parecida a la papa huairo. Esta variedad ha desaparecido de la zona.

Papa Cordovina: papa arenosa de forma redonda, de color medio anaranjado, de sabor muy agradable. Esta variedad se halla en proceso de extinción en la zona.

Papa arenosa, de forma aplanada, de color medio negruzco. Esta variedad se halla en proceso de extinción en la zona.

Papa arenosa de forma alargada, de color rojizo jaspeado, variedad desaparecida de la zona

Papa blanca de forma alargada, no arenosa, adecuada para la elaboración del chuño. Variedad desaparecida.

Papa en forma de mano de gato, arenosa. También esta variedad ha desaparecido de la zona.

Variedad de papa amarilla que está en vía de extinción. 


\section{Siri}

Papa blanca, de forma alargada, especial para la elaboración del chuño. Esta variedad ha desaparecido de la zona.

En la canción Sillkaw -que, como se ha dicho, también es un diálogo entre un coro de hombres y otro de mujeres- los hombres representan a un padre y las mujeres a una madre. En esta canción se expresa el deseo de que sus hijos adquieran una profesión como la de maestro o maestra de escuela, enfermera, abogado, médico o arquitecto:

$$
\begin{array}{cc}
\text { H Ana wawallayki, } & \text { Tu hija Anita, } \\
\text { sillkaw, sillkaw, } & \text { sillkaw, sillkaw, } \\
\text { Manam imapaqchu, } & \text { no es para nadie, } \\
\text { sillkaw, sillkaw, } & \text { sillkaw, sillkaw, } \\
\text { Carlos churiypaqmi,. } & \text { Es para mi hijo Carlos, } \\
\text { sillkaw, sillkaw. } & \text { sillkaw, sillkaw. }
\end{array}
$$
M. Manam wawaytaqa, No a mi hija, sillkaw, sillkaw. sillkaw, sillkaw.
Mayistrapaqmi, sillkaw, sillkaw. sillkaw, sillkaw.
Ducturapaqmi, sillkaw, sillkaw. sillkaw,sillkaw.
Abugadapaqmi, sillkaw, sillkaw.
Arquitectupaqmi, sillkaw, sillkaw.

Estas aspiraciones revelan, de un lado, que la condición de campesino, consustancial a toda sociedad agraria, ha sufrido un grave deterioro como categoría socioeconómica, y ésto sólo puede explicarse por la diferenciación campesina, consecuencia de la existencia de la propiedad privada de tierras en el seno de la comunidad, lo que ha hecho que existan campesinos pobres; y, de otro lado, revelan también la convicción de que su lengua y su cultura son un obstáculo ante los patrones de vida que ha establecido el orden dominante, es decir la cultura occidental y su vehículo de expresión en esta parte el mundo, el idioma castellano.

\section{LASCANCIONES}

$$
\text { IRA }
$$$$
\text { I }
$$

\section{M Ñachumirakichasqaina, sewanaykulllay, kunyaq papa yayakmanpaq, sawanaykullioy.}

\section{H. Nam irakichasquĩa sawanay kullloy, kumyaq papayaykumanpaq sewanay kulllary.}

\section{¿Yaestálacrapreparada, sawrayklalllay, peraquectire lapapakunyaq sawanay kulliay?}

$$
\begin{aligned}
& \text { Yaestálacrapreparada, } \\
& \text { sewanaykulllay, } \\
& \text { paraqueentre la papalunyaq, } \\
& \text { sawanaylulllary. }
\end{aligned}
$$

M Ñachumirakichasquina, sawanaykillary, kurdubini. papa yaykunanpeq, sawanay kullkury.

H. Ñam irakichasqaĩa, sawanaykullkuy, kurdubina papa yaykunanpaq, sawanaylaillary.

M Ñachumirakichasqaĩa, sawanay kullkary, yana paltas papa yaykunanpaq. sawanaykullkuy

\section{H Ñam irakichasqñ̃a sawanaykullkyy, yana paltas papa yaykunanpaq, sawanaykullky.}

\section{M Ñachum irakichasqaña} sawanaykullkuy, aqu suytu papa yaykunanpeq, sawanay kulllouy.

H Ñam irakichasqaña sawanay kulllary, aqu suytu papa yaykunanpeq, sawanaykullkwy.

¿Yaestálacrapreparada sawanay kullhury,

para que entre la papa cordovina, sawanaykullary?

Yaestá laerapreparada, sawanaykulliay,

para que entre la papa cordovina, sawanaykulliky. ¿Yaestála era preparada,
sawanay kullauy,

para que entre la papa yana paltas, sawaray kullkuy?

Ya extálaerapreparada, sawanalaullary,

para que entre la papa yana paltas, sawanay kullkuy.

¿Yaestálaerapreparada sawaraykulllkuy, para que entre la papa aqu suytu, sawanay kullkary?

Yaestála erapreperada, sawanay kulllay, para que entre la papa aqu suytu, sawanay kullikuy.

M Nactumirakichasqeña, sawanay kullhuy, qala suytu papa yaykunanpaq sawanaykullary.

¿Yaestá laera preparada, sawanay kullkuy, para que entre la papa qala suytu, sawanay kullkury.

H Ñamirakichasqaña, swanay kullkuy, qala suytu papa yaykunanpaq, sawanaykullhary.

Yaestálaerapreparada, sawanaykullkry, para que entre la papa qala suytu, sawanay kulllky.

\section{Nactumirakichasquña sowanaykalllayy, misipamakinpapa, yayakumanpeq, sawanay kullkwy.}

H Ñamirakichasqũa sawanaylulllky, mispamakinpapa yaykunapeq, sawanay kullkay.

¿Yaeáálacrapreparada, sawanay kullibyy, paraqueentre lapapamanode gato, sawaraykulllary.

Ya extalacrapreparada, sawanay kulllury, paraqueentre la papamanode gato, sewaraylulllkuy.

M Ñachum irakichasqaĩa, sawrengylulliky, ¿Yaestálacrapreparada, sawanaykalllary, qithurums pepayaylamanpeq, praqueertrelapapaqithunnus, swanaykulliay. sawany kulllkary? 
H. Namirakichasqañ sawanaykullikry. qillururnus papa yayakunarpeq, sawanaykullhary.

M Ñachumirakichasqãna sawanaylullkay, sin papayaykunanpaq sawanaykullkuy

H. Ñamirakichasquñan, sawanaykullkuy, sin papa yaykunanpeq. sawanaykullkury.

II

\section{M ilmasawawankimarraq sawanaykullikuy. barnitinumakykiwan, sawanay kullkuy?}

H. Kananmisawasqayki, sawanaykullkuy, kaballinu usuchata. sawnaykullkry,

M ilmasawawankimarraq sewaraykullkny, yanachumamakiykiwan, sawanaykulkuy?

H. Kananmisawasqayki, sawanaykullkory, qui palmamakichaywan, sawanaykullkary.

III

M ;Aparquy,aparquy,tuyas, aryas!

¡Sawarquy, sawarquy, tuyas, ¡Cárgala, cárgala tuyas, tuyas! uryas!

¡Ñaqcharquy,ñaqchanquy, uryas, ¡Péinala péinala, tuyas, tuyas! uryas!

¡Mucharquy, mucharquy, wyyas, ¡Bésala, bésitla tuyas, tuyas! uryas!

¡Saquarquy,saqarquy, tuyas, ¡Túmbala, túmbala, uryas, tuyas! wyyas!

M ¡Kutichipamuway, tuyas, tuyas! ¡Devuélmela, tuyas, uryas! ¡Apapamuwayña, uyyas, uryas! ¡Yaregresámela, uyyas, uryas!

Tuyas: pájaro de color gris, de pecho y pico amarillo,que acostumbra picotear frutos tiernos, como el choclo, las habas, etc.

\section{SILLKAW}

H Ana wawallayki, sillkaw, sillkaw, manam imapaqchu, sillkaw, sillkaw.

Carlos churiypaqmi. sillkaw, sillkaw.

M Manam wawaytaqa, sillkaw, sillkaw.

Mayistrupaqmi, sillkaw, sillkaw.

Dukturapaqmi, sillkaw, sillkaw.

Abugadupaqmi, sillkaw, sillkaw.

H Rosa wawallayki, sillkaw, sillkaw, manam imapaqchu, sillkaw, sillkaw.

Josey churiypaqmi, sillkaw, sillkaw.

M Manam wawaytaqa, sillkaw, sillkaw.

Infirmi apaqmi, sillkaw, sillkaw.

Abugadapaqmi, sillkaw, sillkaw.

M Carlos churillayki, sillkaw, sillkaw, manam imapaqchu, sillkaw, sillkaw.

Rosa wawaypaqmi, sillkaw, sillkaw.

H Manam churiytaqa, sillkaw, sillkaw.

Abugadupaqmi, sillkaw, sillkaw.

Inginirupaqmi, sillkaw, sillkaw.

Midikupaqmi, sillkaw, sillkaw.

M. Andres churillayki, sillkaw, sillkaw, manam imapaqchu, sillkaw, sillkaw.

Carmen wawaypaqmi, sillkaw,sillkaw.
Tu hija Anita, sillkaw, sillkaw, no es para nadie, sillkaw, sillkaw.

Es para mi hijo Carlos, sillkaw, sillkaw.

No a mi hija, sillkaw, sillkaw.

Es para maestra, sillkaw, sillkaw.

Es para doctora, sillkaw, sillkaw.

Es para abogada, sillkaw, sillkaw.

Tu hija Rosita, sillkaw, sillkaw, no es para nadie, sillkaw, sillkaw.

Es para mi hijo José, sillkaw, sillkaw.

No a mi hija, sillkaw, sillkaw.

Es para enfermera, sillkaw, sillkaw.

Es para abogada, sillkaw, sillkaw.

Tu hijito Carlitos, sillkaw, sillkaw, no es para nadie, sillkaw, sillkaw.

Es para mi hija Rosa, sillkaw,sillkaw.

No a mi hijo, sillkaw, sillkaw.

Es para abogado, sillkaw, sillkaw.

Es para ingeniero, sillkaw, sillkaw.

Es para médico, sillkaw, sillkaw.

Tu hijo Andresito, sillkaw, sillkaw, no es para nadie, sillkaw, sillkaw. Es para mi hija Carmen, sillkaw, sillkaw. 
H Manam churiytaqa, sillkaw, sillkaw.

Arkitiktupaqmi, sillkaw, sillkaw.

Midikupaqmi, sillkaw,sillkaw.
No a mi hijo, sillkaw, sillkaw.

Es para arquitecto, sillkaw, sillkaw.

Es para médico, sillkaw, sillkaw.

\section{BIBLIOGRAFÍA}

\section{BOLAÑ̃os, Sara}

1982 Introducción a la teoría y práctica de la sociolingüística. México D.F.

\section{CERRÓN PALOMINO, Rodolfo.}

1995 Motosidad y enseñanza del castellano. En: Aportes para la enseñanza del lenguaje. INIDE. Lima, 1995.
GÁLVEZASTORAYME, Isabel

"Léxico del quechua de Arma". Serie INVEL No 8. UNMSM.

MONTOYA, Rodrigo (et. al.)

1987 La sangre de los cerros. Urqukunapa yawamin. Mosca Azul. Lima.

\section{SILVACORVALÁN, Carmen.}

1989 Sociolingüística:Teoría y análisis. Editorial Alhambra. Madrid, 1989.

TORERO, Alfredo.

1964 "Los dialectos quechuas".En:Anales cientificos. Vol. II. UNA. Lima.

1974

El quechua y la historia social andina. Universidad Ricardo Palma. Lima. 